\title{
The Dual Role of Evo-Devo Mechanisms and THE EXTENDED EVOLUTIONARY SYNTHESIS
}

\author{
GUILLERMO FOLGUERA \\ Universidad de Buenos Aires - CONICET, ARGENTINA \\ guillefolguera@yahoo.com.ar \\ NiCOLÁS JOSÉ LAVAGNINO \\ Universidad de Buenos Aires - CONICET, ARGENTINA \\ nlavagnino@gmail.com
}

\begin{abstract}
The distinction between mechanisms that generate biological variation and mechanisms that modify it has been important in contemporary Biology, especially since the establishment of the Evolutionary Synthesis (ES) in the first part of the twentieth century. In the ES, and in its subsequent legacy to evolutionary biology, the focus was directed at mechanisms that modify biological variation. In recent years, evo-devo (Evolutionary Developmental Biology) emerged as an area of knowledge that proposes to extend the ES in many forms. In this sense, given that evo-devo integrates different areas of Biology, different types of mechanisms can be found. In order to understand evo-devo mechanisms, as well as its relation with the ES, we analyzed the role that evo-devo mechanisms play with respect to biological variation. The main question in our analysis was: do evo-devo mechanisms have a function of generators and/or modifiers of biological variation? We focused on three evo-devo mechanisms: environmental induction, hypervariability/somatic selection and developmental bias. Our analysis showed a different characterization of the action of evo-devo mechanisms. This heterogeneity in the role of evo-devo mechanisms shows that, in general, the distinction is maintained but there is a mechanism that presents a dual role. Our analysis indicates that, at least with respect to mechanisms, evo-devo extends and departs from what was proposed in the evolutionary synthesis.
\end{abstract}

Keywords: Evo-devo • biological mechanisms • biological hierarchies $\bullet$ mechanisms that generate biological variation $\bullet$ mechanisms that modify variation

RECEIVED: 20/06/2017 REVISED: 03/11/2017 ACCEPTED: 09/05/2018

\section{Generating and modifying biological variation and evo-devo mechanisms}

Genetics of Populations, Evolutionary Ecology, and Classical Genetics are the areas of knowledge of the Evolutionary Synthesis (hereinafter ES) that deal with mechanisms of evolution (Arthur 1997). One of the most important conceptual distinc- 
tions within this group of theories relates to the differentiation between mechanisms that generate biological variation (hereinafter $M g s$ ) and mechanisms that modify it (hereinafter $\mathrm{Mms}$ ). In this sense, Classical Genetics researchers have proposed a great number of mechanisms that generate variation, which are known as "mutation in a broad sense" and include: mutation, recombination, macro-deletions, duplications, inversions, hybridization, etc. (Endler and McLellan 1988). On the other hand, Genetics of Populations mainly generated models to describe the action of mechanisms that only modify variation: natural selection, mutation, gene flow and genetic drift (see, for example, Templeton 2006, Hedrick 2011).

However, this classification between Mgs and Mms has some important exceptions. On the one hand, we acknowledge that in the canonical version of the ES, mutation exhibits certain peculiarities, since it is considered that in situations where there are low mutation rates it acts only as an $M g$, and in situations with high mutation rates it acts as an $\mathrm{Mm}$, though a weak or unimportant one (Fisher 1930; Haldane 1933). We will return to the particularities of mutation as a biological mechanism and its dual role in the next section. Also, in recent years there has been some debate about the "creative role" of natural selection, making this mechanism a candidate to be an $M g$ (see, for example, Forber 2005; Nanay 2005; Martinez and Moya 2009). Unlike the case of mutation, the debate on the classic "destructive role" versus a supposedly neglected "creative role" of natural selection has had different characteristics than the ones proposed in the distinction made by the ES. Nevertheless, this discussion is another case that points toward complexity in the actions of mechanisms in evolution. In this context, the recovery of the complexity of evolutionary mechanisms is important not only to clarify the status of the different evolutionary mechanisms that diverse areas of knowledge propose (either from the classic areas of knowledge within the ES or from others), but also because it is a possible path to understand the type of contributions that each area of knowledge makes to evolutionary biology.

The ES was the theoretical system accepted and used to account for evolutionary phenomena between the 1930s and 1970s. Since then, the ES was criticized for having problems in dealing with several phenomena, like molecular, macroevolutionary and ontogenetic phenomena. Among the identified difficulties of the ES appears the fact that it has proposed only a few mechanisms - the ones mentioned earlier to deal with evolutionary phenomena. For example, Amundson says that, "[w]hen Synthesis theorists speak of "evolutionary mechanisms" they mean population genetic processes, usually involving selection (but possibly drift or other parameters)" (Amundson 2005, p.167). Since then there has been agreement on the necessity of extending the ES and also different attempts to succeed in doing so (just to name a few examples, see Stanley 1975; Gould 1982; Buss 1983; Jablonka 2006; Müller 2007; Pigliucci 2007; Pigliucci and Müller 2010). One of the areas of knowledge often mentioned as important in the extension and modification of the ES is Evolu- 
tionary Developmental Biology (hereinafter evo-devo) (Müller 2007; Pigliucci 2007). Evo-devo has presented different research programs (Laubichler 2007; Müller 2008). However, within this heterogeneity it is clear that one of its main objectives is the integration of the study of the evolution and development of organisms (Love 2003). The extension to the ES that evo-devo proposes takes many forms. To name a few: the consideration of development not only as a buffer for genetic variation but also as a direct actor in the generation of evolutionary novelties where non-genetic properties of development (epigenetic) are relevant (Muller 2007); the importance of modularity and phenotypic plasticity as causes in the evolution of organisms (Muller 2007); the consideration of a creative role of the environment as co-responsible for the evolution of phenotypes (Gilbert and Epel 2009; Odling-Smee 2010) in addition to its classic characterization as filter; and, more in line with the theme of this work, the proposal of other mechanisms, rather than natural selection, as responsible for evolutionary directionality (Arthur 2002; Muller 2007).

In this context, in order to understand in what sense the ES has been extended, how evo-devo has been involved, and also what new challenges must be faced in the coming years, herein we analyze whether evo-devo's mechanisms act as Mgs or as $M m s$, or if they are mechanisms that can act in both ways and hence have a dual role. As will become clear in the following sections, we address the roles of evo-devo mechanisms with respect to biological variation in terms of the ES categories. We recognize that the most followed strategy to generate an extension to the ES has been by means of altering and changing the fundamental categories and conceptualizations of this theoretical system. Undoubtedly, this strategy is extremely useful and necessary because it provides strategies capable of incorporating the theoretical, phenomenological, and ontological diversity that was generated in the last decades. In this line, Brigandt points out that "some aspects of evo-devo mandate a revised, broadened philosophical conception of mechanisms and mechanistic explanation" (Brigandt 2015, p.136), and Mc Manus says that this kind of strategy "ultimately seeks to offer an alternative to the current main conceptions of what is a mechanism" (Mc Manus 2011, p.533). Also, this strategy involves denying traditional distinctions as proximate-ultimate causation (Calcott 2013). With regard to this distinction, WestEberhard expresses a common theme within evo-devo: "the proximate-ultimate distinction has given rise to a new confusion, namely, a belief that proximate causes of phenotypic variation have nothing to do with ultimate, evolutionary explanation" (West-Eberhard 2003, p.11). However, there is another type of strategy that still needs to be executed: analysis of the extension to the ES considering its own categories and conceptualizations. This will allow for a better understanding of which are the new analytic and heuristic paths that need to be followed and incorporated, as well as of the boundaries of pre-existing categories. Of course, our position is that these two strategies are not antagonistic, but rather absolutely complementary. 
Given the objective of the paper and the general strategy chosen to achieve it, our main hypothesis is that although the general distinction of mechanisms between generators and/or modifiers of biological variation is maintained in evo-devo, at least one evo-devo mechanism can be recognized that presents a clear and equivalent dual role (i.e., it can act consistently both as an $\mathrm{Mg}$ or as an $\mathrm{Mm}$ ). This situation represents a partial extension and departure from what was proposed in the ES for the role of mechanisms with respect to biological variation.

To address our hypothesis, the present paper is organized as follows. In the second section, we will highlight some general ideas about mechanisms in the ES and in its extended and hierarchical version. Then, we will briefly characterize the two types of mechanisms that operate in Biology: mechanisms that generate and mechanisms that modify biological variation. In the third section, we will analyze, following our hypothesis, three mechanisms that were proposed within evo-devo. Finally, on the basis of previous considerations, in the last section we will present some general conclusions and a discussion.

\section{Searching for the criteria to recognize mechanisms that modify and/or generate biological variation}

\subsection{Initial considerations about the analysis of mechanisms}

At first, it is important to recognize that we are following the implied notion of mechanisms in the canonical version of the ES. Even if the architects of the ES did not explain it or make it explicit, for this theoretical construction it was clear what a mechanism did. Templeton states it as follows: "Population genetics has always played a central role in evolutionary biology as it deals with the mechanisms by which evolution occurs within populations and species, the ultimate basis of all evolutionary change" (Templeton 2006, p.ix). The analysis of evo-devo mechanisms is based on this notion. We also use a distinction present in the ES about mechanisms' action; there are those that modify variation (Mms) and others that originate it (Mgs), giving rise to the possibility of a dual role. This distinction, as was stated in the previous section, is a factual situation in the ES. It is precisely from these categories and conceptualizations of the ES that we will try to extract the criteria to analyze the mechanisms suggested in evo-devo.

Secondly, it is important to note that our analysis of evo-devo mechanisms presents another fundamental aspect that should be considered and that is often ignored when considering the theoretical core of the ES. This additional complexity refers to one of the characteristics of the proposal for an Extended Evolutionary Synthesis (hereinafter EES) suggested in recent decades: the notion of a hierarchy in evolu- 
tionary biology together with its multi-level structure (see, for example, Gould 1980; Gould 1982; Vrba and Gould 1986). Moreover, the EES proposed a general mechanism, named selection. For example, in Lieberman and Vrba's words, "selection is the interaction between heritable, varying, emergent characters of individuals and the environment that causes differences in birth and/or death rates of those individuals" (Lieberman and Vrba 1995, p.394). Selection was, hence, conceptualized as a general mechanism of which natural selection is only a particular case. In recent years, many researchers have favored the incorporation of a general selection mechanism, acting at different levels, into the conceptualization of the evolutionary processes (Jablonski 2000; Kerr and Godfrey-Smith 2002; Shelton and Michod 2010; Wilson 2010). This is known as "multi-level selection theory", and it is recognized for its high "potential for empirical and theoretical advances" (Jablonski 2010, p.344) and claimed to be "essential to understanding long-term evolutionary processes" (Jablonski 2010, p.350). Thus, multi-level structure and hierarchy within Evolutionary Biology will be taken into account in our analysis of evo-devo mechanisms.

At this point, having settled the last considerations, it is important to remember that the general question in our analysis is whether evo-devo mechanisms have a dual function as generators and modifiers of biological variation. To this end, in the next section we clarify the criteria adopted to recognize whether evo-devo mechanisms modify and/or generate biological variation.

\subsection{Mechanisms that modify biological variation}

From an ES perspective, Mms are considered mechanisms that modify the frequencies of genetic variants (genetic variation) between two generations for a particular population. It is important to note here that there are different scenarios where there are no absolute changes but there are evolutionary mechanisms acting in a compensatory way. Given the considerations made in the previous section, it cannot be ignored that evolution presents a multi-level structure, thus the search for general conditions to identify Mms and Mgs must incorporate a hierarchical perspective. In this sense, even if the ES proposed different mechanisms to explain variational changes over time, it is clear that from a hierarchical perspective the conditions to be met in order to consider that a mechanism acts as an Mm could not be restricted to the proposals of the ES. This would result in including only those mechanisms involved with genetic variation at microevolutionary levels. Also, this definition may present numerous problems, two of which could be easily identified. First, the notion of a "generation" is very clear in the case of a population (at least for a great number of vertebrate organisms), but it is not so evident for all evolutionary levels. For instance, when analyzing the species level, what does "one generation" mean? In any event, what is at stake is biological change through time, regardless of how 
biological entities at different levels pass through time. Second, in the case of the microevolutionary proposal within the ES, the population is assigned the evolutionary role of being the unit of evolution; in a hierarchical proposal it may be necessary to detect an analogous spatially and temporally defined entity for each one of the levels of the hierarchy. The problem lies in that while it is easily designated in the case of microevolution, it presents an imprecise status for other levels; i.e., which is the unit of evolution when dealing with change between species? Given that conceiving of units of evolution from a hierarchical point of view is not an easy or trivial task, we will use the general term domain. This term represents any spatially and temporally defined entity where evolutionary change happens at any level, with population (the microevolutionary unit of evolution) being a special case of a domain. Therefore, a more general criterion which enables the recognition of $M m s$ at different levels of the evolutionary dimension must be employed. Thus, from a hierarchical perspective, Mms could be defined as those mechanisms that modify the frequencies of the types of biological entities (some kind of biological variation) in a domain for a particular interval of time.

Since this definition may present operative problems when trying to search for biological mechanisms that fit into it, we decided that in order to identify Mms we will follow an alternative path and propose that hierarchical $\mathrm{Mms}$ are those that, as a consequence of their action, produce a biological phenomenon that is considered an evolutionary event in the sense of the canonical version of the ES. As a direct outcome, it is necessary to define which are the requirements that any mechanism must satisfy to produce a biological phenomenon that is an evolutionary event in the sense of the ES (and consequently be considered as an $\mathrm{Mm}$ ). We recognize at least three requirements that should be taken into account:

\section{Minimum number}

This requirement is motivated by Caponi's (2008) proposal. It refers to the idea that an evolutionary event, in the sense of the ES, must be composed of (at least) two different biological types, and both (or all) with a number of representatives extensively greater than one. In terms of the action of mechanisms, it implies that, to be considered an $\mathrm{Mm}$, any mechanism must produce (in the particular domain defined for the level of biological organization where the mechanism acts) at least two different biological types, and both (or all) with a number of representatives greater than one. As we can see, the problem of the diffuse boundary of the aforementioned domain also becomes tangible here.

What is the relevance of this requirement? Its importance lies in that it is the first step to differentiate $M g s$ from $M m s$, being that the results of the action of $M g s-\mathrm{a}$ temporally and spatially unique biological event — should not be considered as an 
evolutionary event in the sense of the canonical version of the ES. However, as we will see, the minimum number requirement is necessary but not sufficient.

\section{Repeatability}

Prior to the recent proposal of an EES, the requirement of a steady repetition of biological variants trough time was exclusively guaranteed by means of genetic heredity. However, in recent years, the number of ways that ensure the repetition of a particular biological attribute over time has increased (see, for example, Jablonka et al. 1998; Jablonka and Lamb 2010). In particular, we consider at least three different types of repeatability: genetic heredity (repetition of a biological trait through time due to the transmission of information by means of nucleotide sequence), broad-sense heredity (repetition of a trait through time by means of another unit of information rather than the genetic one, as, for example, epigenetic) and recurrence (a biological trait is consistently originated through time but not due to the transmission of internal information - whether genetic or epigenetic - but as consequence of the steady repetition of another factor, for example, a similar environmental effect on a phenotype of any biological entity that repeats through time). As is obvious, this criterion guarantees one of the necessary aspects of an evolutionary event in the sense of the canonical version of the ES: its permanence throughout the passage of biological entities over time within a domain for a particular level. The repeatability requirement is also necessary but not sufficient.

\section{Bias}

The criterion of bias is inspired by Yampolsky and Stoltzfus's (2001) population genetics models concerning the requirements to be met by mutation for it to act as a mechanism that modifies biological variation. In general terms, it is based on the differential probability that a biological entity has of turning from a particular state to its inverse (Figure 1a). When this requirement is met, the action of mechanisms changes frequencies of biological entities in a given direction over time (Figure 1b1,2). This requirement guarantees avoiding scenarios with a high number of changes that do not produce any change in the global biological variation (Figure $2 \mathrm{~b} 1,2$ ), due to similar probabilities of turning from one biological state (variant) to its inverse (Figure 2a). As in the cases of minimum number and repeatability, the bias requirement is necessary but not sufficient.

We recognize that ES thinkers considered mutation as an $\mathrm{Mm}$ when it acts in high rates, even in the absence of bias. However, to evaluate the mechanisms of evo-devo we took a different approach, perhaps more restrictive (or hard), and include bias as one of the requirements to be met by any evo-devo mechanism to be considered as 
an $\mathrm{Mm}$. This decision was taken to prevent evo-devo mechanisms considered as Mms from falling into the category of unimportant or weak evolutionary mechanisms, as happens with mutation in the ES (Fisher 1930; Haldane 1933).

a

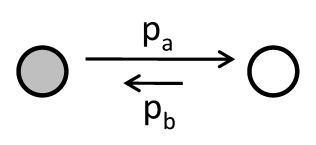

$p_{a}>p_{b}$

b1

b2
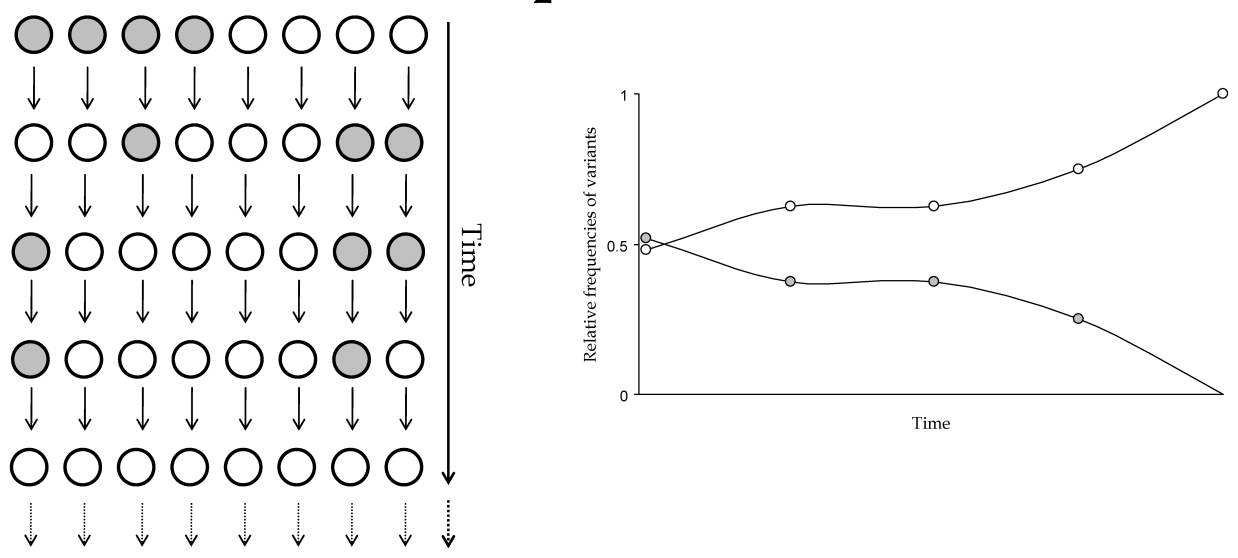

Figure 1: Changes in variation through time due to the action of hierarchical mechanisms that produce bias. (a) As a consequence of the action of the mechanisms, the probabilities of turning from a particular state to its inverse are different. In the example, each circle represents a unitary biological item of any domain and the probability of turning from the "gray variant" to the "white variant" is larger than the inverse. (b1) Simplified representation of the change of a hypothetical biological domain through time when the mechanism involved produces bias. Different colors of biological entities are different variants. (b2) Change of relative frequencies of variants in the presence of bias. Note that there is important and consistent modification of variation through time.

Following the description of the requirements that a mechanism must meet to be considered an evolutionary event in the sense of the canonical version of the ES, it is clear that these requirements have different roles in our analysis. In this sense, bias can only take place if the mechanism at stake previously fulfills the requirements 
of minimum number and repeatability. Thus, bias is a subsidiary requirement of the principal requirements of minimum number and repeatability.

\subsection{Mechanisms that generate biological variation}

When trying to clarify the criteria for recognizing mechanisms that act as biological variation generators (Mgs), once again, it is not easy to establish a general criterion suitable for the different levels of a hierarchy. From an ES perspective, classical genetics provides a great number of $M g s$. In this context, $M g s$ are those mechanisms that generate any type of genetic variant that did not exist previously (de novo genetic variation) in a particular population from one generation to the next. In this sense, in addition to the consideration of de novo generation of biological variation in an ES sense, we must include innovation and novelty as different cases of generation of biological variation with respect to the case mentioned in the ES. There are several conceptualizations of novelty and innovation within evo-devo as part of the EES. Two common features of these conceptualizations are that they include a hierarchical perspective of the origin of de novo biological structures and/or functions, a clear reference to the inclusion of macroevolutionary levels; and that the changes involved are qualitative, rather than quantitative as in the ES (Müller and Wagner 1991; Love 2006; Pigliucci 2008; Müller 2010; Brigandt and Love 2012). Following the strategy of analysis presented in the introduction, and as will become clear below, the notion of $\mathrm{Mg}$ presented include both conceptualizations of de novo generation of biological characteristics.

As in the case of Mms, if evolutionary biology is conceived of from a hierarchical perspective, the microevolutionary definition of $M g$ may present several problems; two of them are those previously described for $M m s$, and in this case one more is added. This additional problem with $M g s$ has to do with the need to establish a clear criterion to determine what a variant is. In a hierarchical definition, each level should present a particular notion of a biological variant, which in most cases may differ or be broader than a strict microevolutionary notion. Thus, a clear criterion to determine what a biological variant is must be established. In general terms, this may be the least problematic issue that arises when using the hierarchical definition of $M g$, because the notion of a biological variant is present, more or less explicitly, within each area of knowledge that studies different levels. Therefore, from a hierarchical perspective, and after considering the two problems outlined above and the additional $M g$ difficulty, $M g s$ could be understood as those mechanisms that act through time generating a particular variant that did not exist previously in the domain of the level being analyzed.

As in the case of Mms, the operative problems of trying to search for biological mechanisms that act as established by the hierarchical definition of $M g$ led us to 
a

$$
\begin{aligned}
& \underset{p_{b}}{\stackrel{p_{a}}{\rightleftarrows}} \bigcirc \\
& p_{a}=p_{b}=0.5
\end{aligned}
$$

b1

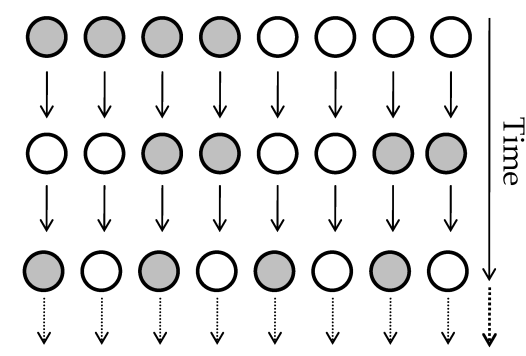

b2

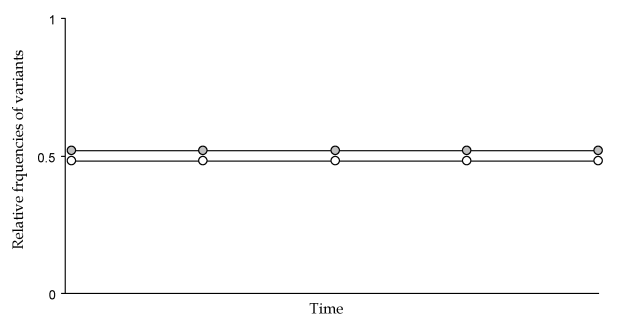

Figure 2: Changes in variation through time due to the action of hierarchical mechanisms that do not produce bias. (a) As a consequence of the action of the mechanisms, the probabilities of turning from a particular state to its inverse are equal. In the example, each circle represents a unitary biological item of any domain and the probability of turning from the "gray variant" to the "white variant" is the same as the inverse. (b1) Simplified representation of the change of a hypothetical biological domain through time when the mechanism involved does not produce bias. Different colors of biological items are different variants. (b2) Change of relative frequencies of variants in the absence of bias. Note that there is not a consistent change of biological variation through time

consider a different way of recognizing them. This alternative proposal is derived from what has been said of the requirements that a mechanism must meet to be considered an evolutionary event in the sense of the canonical version of the ES. In this context, we propose that any mechanism behaves as a hierarchical $M g$ if, as a consequence of its action, the minimum number, repeatability and bias requirements are not simultaneously met.

To illustrate the last point, we can analyze the aforementioned case of mutation in situations where there are low mutation rates. In these scenarios, this mechanism does not meet the principal requirement of minimum number and, as proposed by Yampolsky and Stoltzfus (2001), it produces no bias; but considering the nature of 
mutation, this mechanism always fulfills the principal requirement of repeatability. Therefore, when mutation acts at low mutation rates the three requirements cannot be met simultaneously, so in this scenario mutation acts as an $M g$. In broader terms that also include innovation and novelty, when mechanisms act as hierarchical Mgs they only generate new variants trough time (Figure 3a). They do not drive evolution, as there is no constant drive of biological variation through time as a consequence of their action (Figure $3 b){ }^{1}$

a

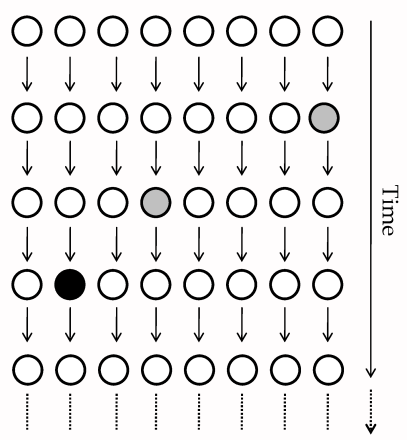

$\mathrm{b}$

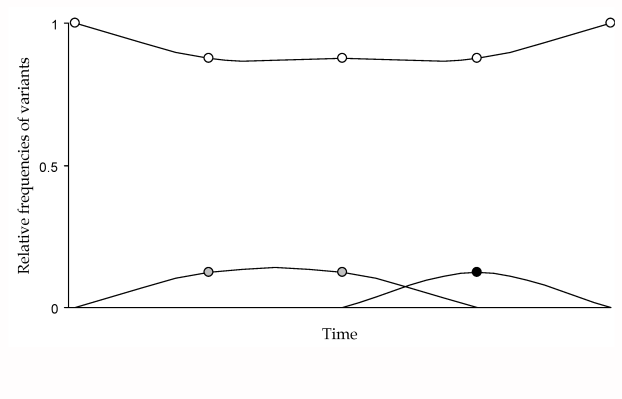

Figure 3: Changes in biological variation through time due to the action of hierarchical mechanisms that generate variation (hierarchical Mgs). (a) Simplified representation of change of hypothetical biological entities through time when hierarchical Mgs occur. Each circle represents a unitary biological entity of any domain. Different colors of biological entities are different variants. (b) Changes in the relative frequencies of variants as a consequence of the action of a hierarchical $M g$. Note that there is not a consistent modification of variation through time.

In the next section, we will analyze three evo-devo mechanisms: somatic selection, environmental induction and developmental bias. In each case, we will describe the mechanism, present documented examples of its action in nature, and then analyze whether they have the potential to act as $M m s$ and/or Mgs.

\section{Evo-devo mechanism analysis}

\subsection{Hypervariability/somatic selection}

\section{Mechanism description}

This mechanism functions in two stages. First, a large number of random variants, modifications, movements or positions of any biological trait are produced, which is 
known as hypervariability. Then, some variants persist while the remainder are eliminated or unoccupied, producing somatic selection. The action of this mechanism is strongly influenced by the environment and it occurs without central coordination, i.e., hypervariability does not have any direct genetic causes but rather is the result of the inherent interactive properties of the materials that form life (West-Eberhard 2003). As a consequence of these properties, this mechanism produces a relatively large array of local biological variants in short periods of time. An early documented example of the action of this mechanism is plant circumnutation, described in 1880 by Darwin in The Power of Movement in Plants, and it refers to erratic exploratory growth movements of stems, roots and leaves that continue insofar as an event or stimulus directs them. Darwin described stem searching movements toward light by marking them on a horizontal glass (example and figure are cited and reproduced in West-Eberhard 2003, p.39). Also in West-Eberhard (2003), two additional examples of hypervariability followed by somatic selection are presented: nervous system organization and vertebrate immune system action.

\section{Hypervariability/somatic selection as $\mathrm{Mm}$}

Since this mechanism could produce a relatively large array of variants of local range, several new variants could be produced at any given moment in the history of a biological entity, each with a near-to-zero probability of being produced and of being almost unique. This is represented in Figure $4 \mathrm{a}$ as a probability of $1 / n$ of producing any variant from a preexisting one, $n$ being the number of variants and, in this case, a large number of variants. Thus, since the probability that any of these new variants is represented in some organismic structure - the domain of action - with a number greater than one is near to zero, this scenario is in disagreement with the minimum number requirement. Also, if each one of the variants produced is almost unique, then all variants should have roughly the same small probability of being produced and it would be almost impossible that any given variant originates at two different moments through time, and therefore this mechanism cannot produce any kind of repeatability (Figure 4b1). Due to its failure to meet the repeatability and minimum number requirements, hypervariability/somatic selection cannot act as an $\mathrm{Mm}$. It could be alleged that the bias requirement could be met if the probability of producing a new variant is lower than that of returning from a new local biological variant to the wild type, which seems to be the case; but since for our analysis of Mms the bias requirement only makes sense if the other two requirements are previously met, this ceases to be important. 
a

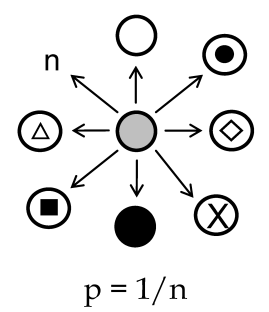

b1

b2
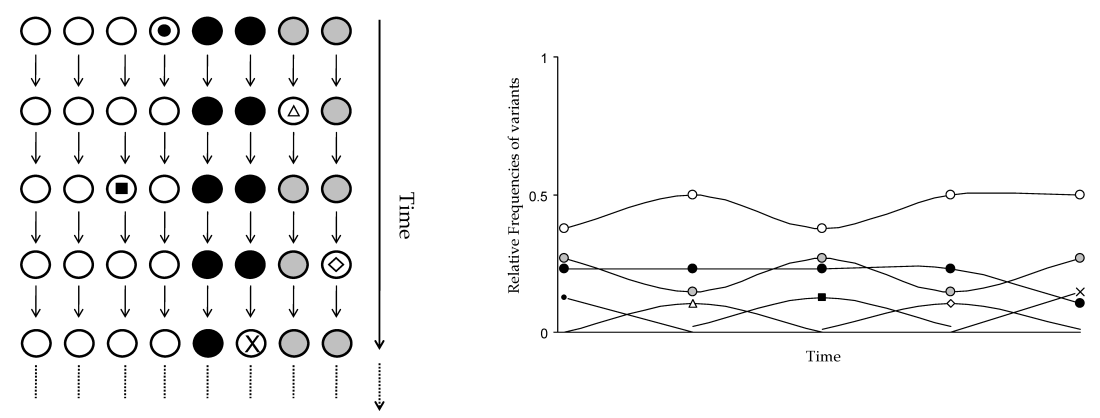

Figure 4: Changes in variation through time due to the action of hypervariability/somatic selection. (a) As a consequence of the action of the mechanisms, a relatively large array of variants of local range are produced and the probability of generating any of the variants from a preexisting one is $1 / n, n$ being the number of variants and a large number. In the example, each circle represents a particular phenotypic variant. (b1) Simplified representation of the change of hypothetical biological items through time when hypervariability/somatic selection acts. Each circle represents a unitary biological item. Different colors and forms of biological items are different phenotypic variants. (b2) Changes in the relative frequencies of variants as a consequence of the action of hypervariability/somatic selection. Note that there is no relevant and consistent change of variation through time, since most new phenotypic variants drop to a relative frequency of zero immediately after being originated

\section{Hypervariability/somatic selection as Mg}

Since as a consequence of its action this mechanism cannot meet the minimum number, repeatability and bias requirements simultaneously, we propose that this mechanism can act as an $\mathrm{Mg}$. Moreover, based on the description of the mechanism and the examples observed in nature, it becomes clear that through its action, hypervariability/somatic selection generates a relatively large collection of previously nonexistent biological variants (Figure 4a, b1). 


\section{Partial conclusions about the hypervariability/somatic selection mechanism}

This mechanism failed to meet the repeatability and minimum number requirements, thus it cannot act as an $\mathrm{Mm}$ even if it meets the bias requirement. Instead, it clearly fulfills the requirements to act as an $M g$, generating previously nonexistent biological variants as a consequence of its action. In this case, the mechanism occurs within the organism domain, at the tissue or organ level. As can be observed in Figure 4, as this mechanism acts, there are many new variants produced at any given time, and none of these new biological types are very likely to occur with a number greater than one. Therefore, hypervariability/somatic selection is not a mechanism that can have the dual role of generating biological variation and driving variational changes.

\subsection{Environmental induction}

\section{Mechanism description}

In this case, the environment acting as an input affects a pre-existing phenotype, driving a change or reorganization that generates a phenotypic variant that is qualitatively different from pre-existing phenotypes and new, i.e., that previously did not exist (Figure 5b1). If the input is recurrent, a group of individuals with the new phenotype is generated. This mechanism is well described in West-Eberhard (2003) and considered important on several occasions, as, for example, in Griffiths and Gray (2004), Jablonka (2006) and Jablonka and Lamb (2010). There are numerous documented examples of environmental induction. The yeast Candida albicans switch from normal yeast cell morphology (white) to an elongated cell type (opaque), which is the mating-competent form of the fungus, induced by anaerobic environmental conditions (Ramirez-Zavala et al. 2008). In both a native and newly established population of finches, breeding females exhibit a novel phenotype in ovulation sequence related to a population-specific environmental stimulus. In the new population, the novel phenotype is induced by a novel environment during females' first breeding and is subsequently retained across breeding attempts. In the native population, the phenotype may also be originated by a novel environmental input and seems to be maintained by recurrent environmental stimulus (Badyaev and Oh 2008). West-Eberhard (2003) cites two more examples: one originally described by Wilson in 1984, where the sea urchin (Sphaerechinus granularis) that bred in an environment without calcareous material or with a small excess of potassium lacks the feeding structures typical of this species, which are present when larvae are bred in an environment rich in calcareous material; and the other proposes that seasonal polyphenism in butterflies originated by environmental induction. 
a

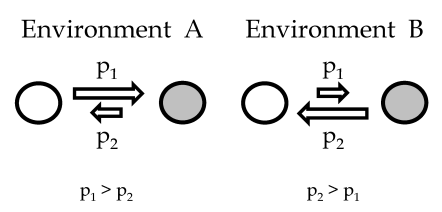

b1 $\mathrm{b}_{2}$
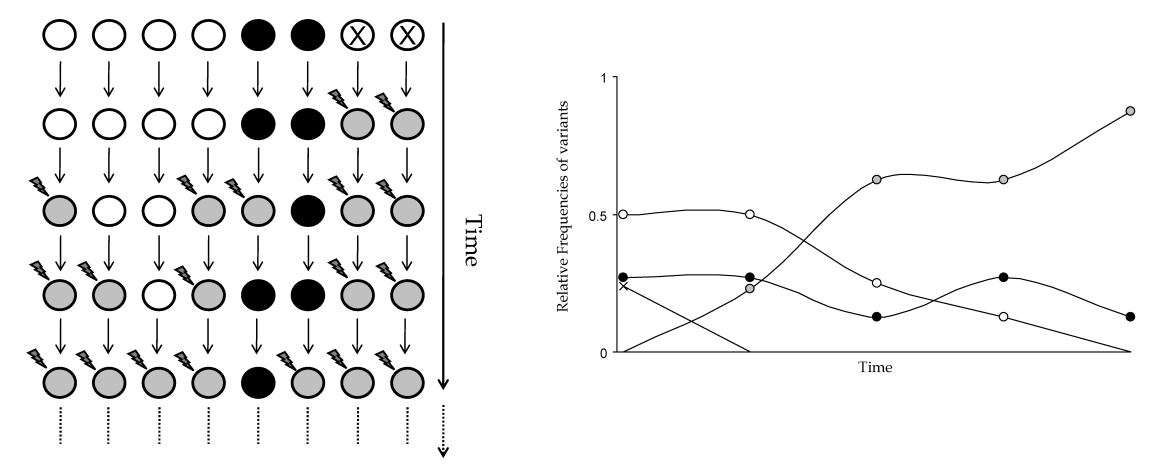

Figure 5: Changes in variation through time due to the action of environmental induction. (a) As a consequence of the action of the mechanisms, the probabilities of passing from a particular phenotypic variant to its alternative are different in different environments. In the example, each circle represents a particular phenotypic variant and the probabilities of passing from the "gray phenotype" to the "white phenotype" are inverse in environments A and B. (b1) Simplified representation of the change of hypothetical biological items through time when environmental induction acts. Each circle represents a unitary biological item. Different colors and forms of biological items are different phenotypic variants. The "lightning" represents the environmental input promoting the induction. (b2) Changes in the relative frequencies of variants as a consequence of the action of environmental induction. Note that the possibility of a relevant and consistent change of biological variation through time is guaranteed

\section{Environmental induction as $\mathrm{Mm}$}

This mechanism can explain the apparition of large amounts of variants in a short interval of time, given that it produces several different biological types with a number of representatives extensively greater than one as a consequence of its action (Figure 5b1). This was previously pointed out by Jablonka (2006) and Griffiths and Gray (2004), and is in agreement with the minimum number requirement. Also, due to the 
characteristics of the mechanism discussed, the probability of generating one phenotypic variant or its alternative could be different in different environments (Figure $5 a)$. This situation is considered as bias and it produces a change in global variation due to dissimilar probabilities of turning from one biological state (variant) to its inverse (Figure 5b2). Finally, repeatability can be achieved by environmental induction in two ways: (i) recurrence over time of the environmental conditions responsible for the induction, which has been proposed by West-Eberhard (2003, p.144); and (ii) by means of broad-sense heredity in the form of heritable epigenetic variation originally induced by an environmental stimulus which does not need recurrence of the environmental condition (Jablonka and Raz 2009; Jablonka and Lamb 2010). Since it fulfills all three requirements posed, environmental induction can act as an $\mathrm{Mm}$.

\section{Environmental induction as $\mathrm{Mg}$}

Given the nature of this mechanism, there are potentially numerous scenarios where the minimum number and repeatability requirements could not be simultaneously met (the bias requirement is subordinated to these two requirements, so in this case its status does not matter); or where minimum number and repeatability requirements could be simultaneously met but the action of environmental induction does not produce bias (when the probability of generating one phenotypic variant or its alternative is the same in different environments). In those cases environmental induction would be acting as an $M g$. Moreover, given that the various examples described previously are just a small sample of the ubiquity of cases where the environment affects a pre-existing phenotype, driving a change or reorganization that generates a phenotypic variant, and that in a number of these cases some of the requirements to produce an evolutionary event in the sense of the ES could not be met, it's rather straightforward to propose the potentiality of environmental induction to act as a mechanism that only generates previously nonexistent biological variants (Figure 5b1).

\section{Partial conclusions about the environmental induction mechanism}

Environmental induction has the potential to satisfy all the requirements we pose must be met to produce an evolutionary event and act as an $\mathrm{Mm}$. Also, given that in some natural situations some of the requirements to produce an evolutionary fact could not be met as a consequence of the action of this mechanism, in these cases environmental induction would only be generating previously nonexistent particular biological variants, i.e., acting as an $M g$. The domain of action of environmental induction is at the level of population or higher. Therefore, environmental induction presents the dual role of generating variation and driving variational changes. 


\subsection{Developmental bias}

\section{Mechanism description}

This mechanism is based on eve-devo's central notion that, somewhere between mutation and selection, development plays a causal role in evolution. Arthur proposed that the link between the supply of variation (new genes or alleles) produced by mutations and the action of natural selection on phenotypes is the developmental system, which is itself always changing. Thus, when mutations occur in genes that control developmental trajectories, they can be more readily altered in some possible trajectories than in others (Arthur 2001a; 2001b; 2002). As Scholl and Pigliucci put it: "the availability of developmental variants co-determines the direction of evolution" (Scholl and Pigliucci 2014, p.7). Positive (developmental drive) and negative (developmental constraint) biases are possible. Developmental bias is related to the degrees of variation in developmental pathways, generating a continuum between highly structured/sparsely variable and loosely structured/highly variable developmental systems (Arthur 2001a; 2001b; 2002) (Figure 6a). Through his research, Arthur documented examples of this mechanism in species of the Lithobiomorpha and Geophilomorpha centipede orders. In the former order, the number of trunk segments, and hence leg pairs, is fixed at 15 for all species. In the latter, the number is variable both within and between species, but all species have an even number of segments. The proposal is that this phenotypic variation is developmentally driven (Arthur 2002).

\section{Developmental bias as $\mathrm{Mm}$}

As can be clearly observed in Figure 6, this mechanism can explain the apparition of at least two biological variants of different types, each one with a number of representatives extensively greater than one in the domain where this mechanism acts, fulfilling the requirement of minimum number. With respect to the bias requirement, Arthur wrote: “... it is clear that developmental reprogramming provides an additional possible source of directionality (...), which might be effective even if the molecular-level changes caused by mutation were entirely random. This directionality lies in the as-yet unknown limitations of developmental systems that permit reprogramming in some directions but result in constraint elsewhere (e.g., the "prohibited" even-segment-number centipedes)" (Arthur 2000, p.55). In this sense, bias is guaranteed, since the probability of turning from one biological state (variant) to its inverse or any other state is not equal (Figure 6a). Lastly, repeatability can be achieved by means of genetic heredity and also by means of broad-sense heredity in the form of any of the types of epigenetic inheritance described by Jablonka and Lamb (2010). 
a

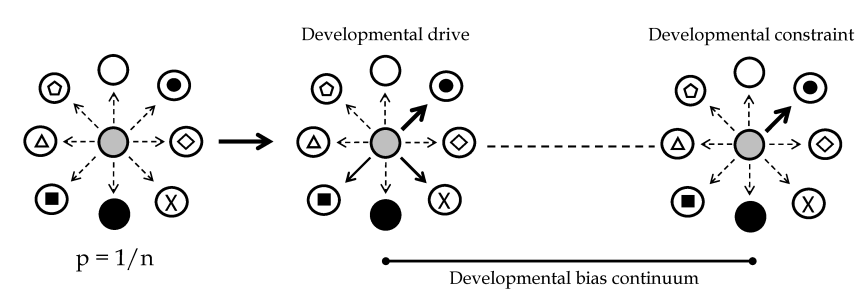

b1

$b_{2}$
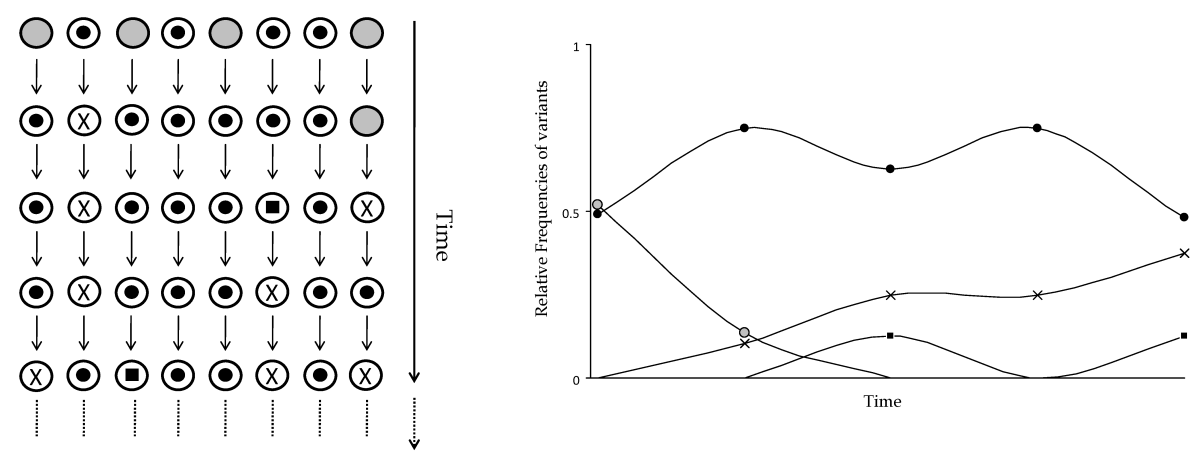

Figure 6: Changes in variation through time in the context of developmental bias. (a) All the potential developmental trajectories (dashed arrows) can be more readily altered in some possible trajectories than in others. This produces an array of possibilities called developmental continuum, developmental drive being the extreme positive bias and developmental constraint the extreme negative bias. (b1) Simplified representation of the change of a hypothetical biological domain through time when developmental drives occur. Each circle represents a unitary biological entity. Different colors and forms of biological items are different phenotypic variants. (b2) Changes in the relative frequencies of variants as a consequence of the action of environmental induction. Note that the possibility exists of a relevant change of biological variation through time 


\section{Developmental bias as $\mathrm{Mg}$}

Given the nature of this mechanism, it seems that when it acts it is not possible for the minimum number, repeatability and bias requirements not to be simultaneously met. That is to say, if developmental bias acts, it always fulfills the three requirements and produces an evolutionary fact - a characteristic shared with, for example, natural selection - and it cannot potentially act as an $M g$. The scenario will be as follows: variation always arises from changes in genes that control development and the action of the mechanism consists only in handling this biological variation, similar to what happens when natural selection acts. By itself, developmental bias does not seem to be able to produce previously nonexistent biological variants; it can only drive its changes in some of the possible established directions.

\section{Partial conclusions about the developmental bias mechanism}

In the case of developmental bias, all the requirements we pose must be met to produce an evolutionary fact are always fulfilled, therefore it only acts as an $\mathrm{Mm}$. The action of this mechanism as a driver of evolution can be appreciated in Figure 6b1,2, where it is clear that when developmental bias occurs, the possibility of a relevant change of biological variation through time is quite certain. On the contrary, we cannot say that, as a consequence of the action of developmental bias, previously nonexistent and particular biological variants are produced. All in all, this mechanism only acts as a modifier of variation that drives variational changes at above-species levels.

\section{General conclusions and final remarks}

Our analysis shows a different characterization of evo-devo mechanisms in terms of their relation to biological variation. Environmental induction is the only evo-devo mechanism that indeed seems to have a dual role of acting as generator and modifier of biological variation, while hypervariability/somatic selection only acts as a generator of de novo variation and developmental bias is only a modifier of biological variation (Table 1). In this scenario, maybe the most interesting case to analyze the action of evo-devo mechanisms with respect to biological variation is environmental induction, a mechanism that presents a dual role of $\mathrm{Mm}$ and $\mathrm{Mg}$. In order to characterize the nature of this dual role, we suggest three possible scenarios to answer the question: in what situations does environmental induction act as an $\mathrm{Mm}$ or $\mathrm{Mg}$ ? At first, two extreme scenarios can be presented. One is when repeatability occurs by means of recurrence of an environmental effect; in this case it is more probable that environmental induction acts as an $\mathrm{Mm}$. This is so because the effect of the mechanism would reach a large part of the biological entities comprising the domain 
involved, thus the apparition and maintenance of different biological types with a number of representatives extensively greater than one is highly probable, whereby the minimum number requirement is most probably met. The other extreme situation is when, as a consequence of the action of an environmental effect, a genetic modification occurs and the environmental input presents no recurrence. In this situation the genetic variant originated is quite probably unique; consequently, environmental induction is acting as an $M g$. There is an interesting intermediate scenario where repeatability is obtained by means of broad-sense heredity in the form of heritable epigenetic variation (once the environmental input ceases). Here, the effect of the mechanism would reach a smaller part of the biological entities comprising the domain than in the extreme recurrence scenario, and a larger part than in the genetic modification scenario. Depending on the scope that the action of the mechanism has by means of heritable epigenetic variation, i.e., the number of representatives of different biological types consistently generated through time, it would be acting as an $\mathrm{Mm}$ or an $\mathrm{Mg}$. Finally, we could ask whether, as in the case of mutation in the ES, the action of environmental induction should be mainly taken as one type of mechanism or the other. If we consider the scenarios for the action of environmental induction proposed, the way that repeatability is achieved is one of the factors that defines whether the mechanisms will act as an $\mathrm{Mm}$ or as an $\mathrm{Mg}$. The possibilities range from recurrence of an environmental input through time without any genetic inheritance involved (environmental induction acting as $\mathrm{Mm}$ ), to a unique event of environmental induction producing a single genetic change that is inherited by means of classic genetic inheritance (environmental induction acting as $M g$ ). In the middle of these two extreme scenarios there is an array of possibilities that involves repeatability by means of broad-sense heredity in the form of heritable epigenetic variation, which does not need repetition of an environmental input nor changes at the genetic level plus genetic inheritance. It does not seem to be evident that one of the extreme scenarios is more likely to happen in nature than the other; and, in any case, the multiple intermediate scenarios could be favorable both for environmental induction acting as an $M m$ or as a $M g$. Therefore, we cannot say that environmental induction acts more consistently as an $\mathrm{Mg}$ or $\mathrm{Mm}$, and the possibility that it fulfills both roles in nature is not unlikely.

In terms of the comparison with the ES and looking at the three evo-devo mechanisms analyzed, altogether it does not seem to be a more relevant role, which would have been the case if all evo-devo mechanisms where characterized only as $\mathrm{Mm}$ or $\mathrm{Mg}$. This represents a differentiation with respect to the ES, where, despite recognizing mechanisms in both categories, the ES considers that the relevant mechanisms are those that modify or drive variation, mainly natural selection. This is clearly stated in a quote by Ernst Mayr where he describes internal differences among ES architects on what evolution was: "evolution continued for the geneticists to be a change of 
gene frequencies, (...) while for the naturalists, evolution was a series of processes resulting in adaptedness and diversity (...)" (Mayr 1993, p.32). Thus, even with differences, ES architects focused in the study of change of biological variation (mainly genetic variation) rather than the study of de novo generation of variation. These shared characteristics, in spite of differences in the way mechanisms act between the ES and evo-devo, are important in the understanding of the type of extension to evolutionary theory implied in the incorporation of evo-devo - particularly in relation to mechanisms, and in particular to the insufficiency in explaining evolutionary phenomena of diverse domains that was pointed out to the ES in recent decades (Amundson 2005). Furthermore, the strategy chosen of using ES categories to understand the action of evo-devo mechanisms is not a question of whether or not we ascribe them to these categories, or to the ES, since they are actually used (Amundson 2005, p.167), but rather a way of approaching the problem of the relationship between the ES and evo-devo indicated by previous authors within the philosophy of biology (for example, see Amundson 2005 and Pigliucci 2008). Our analysis shows that the distinction of $M g s$ and $M m s$ that comes from the canonical version of the ES is maintained in evo-devo, while the ES preference towards Mms and population centrism was not found. Also, moving away from considering evo-devo mechanisms mainly as $M g s$, as is usually done, and considering them as legitimate $M m s$ allows to show that reducing $\mathrm{Mm}$ to the four mechanisms proposed by the ES and giving more importance to natural selection is a problem. This suggests that new lines of investigation are required to overcome these problems.

Heterogeneity in the roles that evo-devo mechanisms play in biological variation shows that the distinction, originally found in the ES, between mechanisms that generate and mechanisms that modify or drive variation is heuristically useful to understand these mechanisms. Also, we propose that heterogeneity in the action of the mechanisms found in our analysis (Table 1) and the need to deepen our understanding thereof in evo-devo are related to the recent origin of evo-devo and its intrinsic diversity and multiplicity of goals (Laubichler 2007; Müller 2008). But beyond this, our analysis points out that, at least with respect to the action of mechanisms, evodevo extends and departs from what was proposed in the ES.

These contributions can be summarized in three aspects. First, it is commonly thought that evo-devo mainly deals with $M g s:$ "(...) the reach of evolutionary theory is expanded in that evo-devo accounts not for what kinds of variation are going to be maintained through natural selection, but also what kinds of variation can possibly arise from specific developmental systems" (Müller 2007, p.947). However, our analysis shows that the contribution of evo-devo to evolutionary biology not only has to do with mechanisms that generate variation where there was none, but also with mechanisms that change or drive biological variation. Second, at least one of the mechanisms of evo-devo, environmental induction, has a dual role of acting as 
Table 1. Summary of the analysis of evo-devo mechanisms. All possible biological scenarios in terms of requirements to produce an evolutionary event in the sense of the canonical version of the ES are shown, as well as expectations in terms of de novo variant origination and variational changes (or stasis) in nature. Table 1 also shows in which scenarios each of the evo-devo mechanisms acts.

\begin{tabular}{|c|c|c|c|c|c|c|}
\hline & & \multicolumn{5}{|c|}{ Mechanism acts as... } \\
\hline & & $M g$ & $M g$ & $M g$ & $M g$ & $\mathrm{Mm}$ \\
\hline \multirow{3}{*}{ 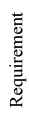 } & Minimum Number & no & yes & no & yes & yes \\
\hline & Repeatability & no & no & yes & yes & yes \\
\hline & Bias & yes & no or yes & no or yes & no & yes \\
\hline & $\begin{array}{l}\text { Possible biological } \\
\text { scenario }\end{array}$ & $\begin{array}{l}\text { High number of unique and } \\
\text { "instantaneous" new variants }\end{array}$ & $\begin{array}{l}\text { Apparition of not unique } \\
\text { "instantaneous" new variants }\end{array}$ & $\begin{array}{l}\text { Low number of new variants } \\
\text { persistent through time }\end{array}$ & $\begin{array}{c}\text { Dynamic } \\
\text { equilibrium }\end{array}$ & $\begin{array}{c}\text { Evolutionary } \\
\text { fact }\end{array}$ \\
\hline \multirow{2}{*}{\multicolumn{2}{|c|}{$\begin{array}{l}\text { EvoDevo mechanisms } \\
\text { action }\end{array}$}} & hypervariability / somatic selection & \multicolumn{4}{|c|}{ Environmental Induction } \\
\hline & & & & & \multicolumn{2}{|r|}{ Developmental Bias } \\
\hline
\end{tabular}

$M g$ : mechanism that generates biological variation. $M n$ : mechanism that modifies biological variation

an $M m$ and an $M g$. Additionally, it does not seem to be given a more relevant role. Thus, the possibility that it fulfills both roles in nature is quite certain. This represents another differentiation with respect to the ES, where mutation is widely recognized to have a dual role, but the $\mathrm{Mm}$ role is minimized as unimportant, leaving mutation fundamentally as an $\mathrm{Mg}$. Finally, we propose that broad-sense heredity, especially epigenetic inheritance, plays an important role in the action of evo-devo mechanisms. The importance of epigenetic inheritance in evolution has been previously treated by many authors (see, for example, Jablonka et al. 1998; Jablonka and Raz 2009; Jablonka and Lamb 2010), but in our analysis it plays a specific role in the action of different mechanisms, and it is another way in which we find an expansion of the ES.

In conclusion, in the present paper we provide a theoretical analysis of evo-devo mechanisms to offer evidence of how evo-devo is providing new conceptual and theoretical elements to evolutionary biology. It is also our understanding that the analysis that we conducted presents some of the many possible ways in which evo-devo is expanding and changing evolutionary synthesis.

\section{References}

Amundson, R. 2005. The Changing Role of the Embryo in Evolutionary Thought: Roots of EvoDevo. Cambridge: Cambridge University Press.

Arthur, W. 1997. The Origin of Animal Body Plans: A Study in Evolutionary Developmental Biology. Cambridge: Cambridge University Press. 
2000. The concept of developmental reprogramming and the quest for an inclusive theory of evolutionary mechanisms. Evolutionary Development 2: 49-57.

- 2001a. Developmental drive: an important determinant of the direction of phenotypic evolution. Evolutionary Development 3: 271-8.

- 2001b. Evolutionary developmental biology: developmental bias and constraint. Chichester: John Wiley \& Sons.

- 2002. The interaction between developmental bias and natural selection: from centipede segments to a general hypothesis. Heredity 89: 239-46.

Badyaev, A.; Oh, K. 2008. Environmental induction and phenotypic retention of adaptive maternal effects. BMC Evolutionary Biology 8: 3.

Brigandt, I. 2015. Evolutionary Development Biology and the Limits of Philosophical Accounts of Mechanistic Explanation. In: P. Braillard; C. Malaterre (eds.) Explanation in Biology: An Enquiry into the diversity of Explanatory Patterns in the Life Sciences, pp.136-74. Netherlands: Springer.

Brigandt, I.; Love, A. 2012. Conceptualizing evolutionary novelty: moving beyond definitional debates. Journal of Experimental Zoology B Molecular and Developmental Evolution 318(B): 417-27.

Buss, L. 1983. Evolution, development, and the units of selection. Proceedings of the Natural Academy of Science USA 80: 1387-91.

Calcott, B. 2013. Why how and why aren't enough: more problems with Mayr's proximateultimate distinction. Biology \& Philosophy 28: 767-80.

Caponi, G. 2008. El segundo pilar. La biología evolucionaria desenvolvimiental y el surgimiento de una teoría complementaria a la teoría de la selección natural. Ludus Vitalis XVI: 3-32.

Endler, J.; McLellan, T. 1988. The processes of evolution: toward a newer synthesis. Annual Review of Ecology Evolution and Systemtics 19: 395-421.

Fisher, R. 1930. The genetical theory of natural selection. London: Oxford University Press.

Forber, P. 2005. On the explanatory roles of natural selection. Biology \& Philosophy 20: 32942.

Gilbert, S.; Epel, D. 2009. Ecological Developmental Biology: Integrating Epigenetics, Medicine, and Evolution. Sunderland: Sinauer Associates.

Gould, S. 1980 The promise of paleobiology as a nomothetic, evolutionary discipline. Paleobiology 6: 96-118.

- 1982. Darwinism and the expansion of evolutionary theory. Science 216: 380-7.

Griffiths, P.; Gray, R. 2004. The developmental systems perspective: organism-environment systems as units of development and evolution. In: M. Pigliucci; K. Preston (eds.) Phenotypic, integration: studying the ecology and evolution of complex phenotypes, pp.409-31. New York: Oxford University Press.

Haldane, J. 1933. The part played by recurrent mutation in evolution. American Naturalist 67: 5-19.

Hedrick, P. 2011. Genetics of Populations. Sudburry: Jones \& Bartlett Learning.

Jablonka, E. 2006. Genes as followers in evolution - a post-synthesis synthesis? Biology \& Philosophy 21: 143-54.

Jablonka, E; Lamb M. 2010. Transgenerational epigenetic inheritance. In: M. Pigliucci; G. Müller (eds.) Evolution-the extended synthesis, pp.137-74. Cambridge: The MIT Press. 
Jablonka, E.; Lamb, M.; Avital, E. 1998. 'Lamarckian' mechanisms in darwinian evolution. Trends in Ecology and Evolution 13: 206-10.

Jablonka, E.; Raz, G. 2009. Transgenerational epigenetic inheritance: prevalence, mechanisms, and implications for the study of heredity and evolution. The Quarterly Review of Biology 84: 131-76.

Jablonski, D. 2000. Micro- and macroevolution: scale and hierarchy in evolutionary biology and paleobiology. Paleobiology 26: 15-52.

- 2010. Origination patterns and multilevel processes in macroevolution. In: M. Pigliucci; G. Müller (eds.) Evolution-the extended synthesis, pp.335-54. Cambridge: The MIT Press.

Kerr, B.; Godfrey-Smith, P. 2002. Individualist and multi-level perspectives on selection in structured populations. Biology \& Philosophy 17: 477-517.

Laubichler, M. 2007. Evolutionary Developmental Biology. In: D. Hull; M. Ruse (eds.) The Cambridge Companion to the Philosophy of Biology, pp.342-60. Cambridge: Cambridge University Press.

Lieberman, B.; Vrba, E. 1995. Hierarchy theory, selection, and sorting. BioScience 45: 394-99.

Love, A. 2003. Evolutionary morphology, innovation, and the synthesis of evolutionary and developmental biology. Biology \& Philosophy 18: 309-45.

- 2006. Taking development seriously: who, what, when, where, why, how? Biology \& Philosophy 21: 575-89.

Martinez, M.; Moya, A 2009. Selección natural, creatividad y causalidad. Teorema 27: 71-94.

Mayr, E. 1993. What was the evolutionary synthesis? Trends in Ecology and Evolution 8: 31-4.

Mc Manus, F. 2011. Development and mechanistic explanation. Studies in History and Philosophy of Biological and Biomedical Sciences 43: 532-41.

Müller, G. 2007. Evo-devo: extending the evolutionary synthesis. Nature Review Genetics 8: 943-9.

- 2008. Evo-devo as a discipline. In: A. Minelli; G. Fusco (eds.) Evolving Pathways: Key Themes in Evolutionary Developmental Biology, pp.3-29. Cambridge: Cambridge University Press.

- 2010. Epigenetic innovation. In: M. Pigliucci; G. Müller (eds.) Evolution-the extended synthesis, pp.307-32. Cambridge: The MIT Press.

Müller, G.; Wagner G, 1991. Novelty in Evolution: Restructuring the Concept. Annual Review in Ecology Evolution and Systematics 22: 229-56.

Nanay, B. 2005. Can cumulative selection explain adaptation? Philosophy of Science 72: 10991112.

Odling-Smee, F. 2010. Niche inheritance. In: M. Pigliucci; G. Müller (eds.) Evolution-the extended synthesis, pp.175-207. Cambridge: The MIT Press.

Pigliucci, M. 2007. Do we need an extended evolutionary synthesis? Evolution 61: 27432749.

- 2008. What, if anything, is an evolutionary novelty? Philosophy of Science 75: 887-98.

Pigliucci, M.; Müller, M. (eds). 2010. Evolution-the extended synthesis. Cambridge: The MIT Press.

Ramirez-Zavala, B.; Reuss, O.; Park Y.; Ohlsen, K.; Morschhauser, J. 2008. Environmental induction of white-opaque switching in Candida albicans. PLoS Pathogens 4: e1000089. 
Scholl, R.; Pigliucci, M. 2014. The proximate-ultimate distinction and evolutionary developmental biology: causal irrelevance versus explanatory abstraction. Biology \& Philosophy 30: 653-70.

Shelton, D.; Michod, R. 2010. Philosophical foundations for the hierarchy of life. Biology \& Philosophy 25: 391-403.

Stanley, S. 1975. A theory of evolution above the species level. Proceedings of the Natural Academy of Science USA 72: 646-50.

Templeton, A. 2006. Population Genetics and Microevolutionary Theory. New Jersey: John Wiley \& Sons.

Vrba, E.; Gould, S. 1986. The hierarchical expansion of sorting and selection; sorting and selection cannot be equated. Paleobiology 12: 217-28.

West-Eberhard, M. 2003. Developmental Plasticity and Evolution. New York: Oxford University Press.

Wilson, D. 2010. Multilevel selection and major transitions. In: M. Pigliucci; G. Müller (eds.) Evolution-the extended synthesis, pp.81-94. Cambridge: The MIT Press.

Yampolsky, L.; Stoltzfus, A. 2001. Bias in the introduction of variation as an orienting factor in evolution. Evolutionary Development 3: 73-83.

\section{Notes}

${ }^{1}$ One last clarification needs to be made. The fact that in the $\mathrm{Mm} / \mathrm{Mg}$ distinction the notion of an evolutionary event, in the sense of the ES, falls on the $\mathrm{Mm}$ side does not mean we consider that $M g s$ have less relevance in evolution. Moreover, since this distinction with its categories and the conceptualization that come from the ES is actually used in evolutionary biology, it becomes irrelevant to the analysis we aim to perform whether or not we think the distinction is correct. As was indicated above, we are using the ES's own categories and conceptualization as a viable strategy to understand evo-devo mechanisms' role in evolution and its relation to the ES.

\section{Acknowledgments}

We thank Bryant Hillas for the revision of the final version of the manuscript and all the members of the Grupo de Filosofía de la Biología (UBA, Argentina) for helpful discussions in early versions of this work. This work was supported by grants from the Universidad de Buenos Aires and the Consejo Nacional de Investigaciones Científicas y Técnicas (Argentina). GF and NJL are members of the Carrera del Investigador Científico of the Consejo Nacional de Investigaciones Científicas y Técnicas (Argentina). 